899625

\title{
ADVANCED RESEARCH DIRECTIONS IN THERMIONIC ENERGY CONVERSION
}

\author{
Elliot B. Kennel \\ WRDC/POOC-2 \\ WPAFB, Oh 45433-6563 \\ Leonard H. Caveny \\ SDIO/T/IS \\ Washington, DC 20301-7100
}

\begin{abstract}
An overview is presented of planned future directions of thermionic energy conversion research for the next decade. The primary focus will be on space nuclear power applications in the ten to thousand $\mathrm{kW}$ range. In addition to refining the state of the art for the conventional ignited mode converter, there are more generic technologies which will be pursued for a wide range of duty cycles.
\end{abstract}

\section{INTRODUCTION}

The successful exploitation of the space resource depends upon efficient, economical, reliable and safe power supplies for future spacecraft of all types. Thermionic conversion is perceived as an important part of the space power picture of the 1990's and beyond because of its well-documented advantages, such as high fraction of Carnot efficiency, high heat rejection temperature, modularity, static operation and so on. Currently there is a dichotomy of interest in the conventional multicell in-core thermionic fuel element (TFE) as well as planar converters compatible with high temperature graphite-based reactors such as the Romashka which was successfully tested in 1964 [1].

It is perceived that initial US requirements for space use of thermionic reactors may emerge in the late 1990's in the low tens of kilowatts range. More ambitious multimegawatt requirements are expected in the post-2000 time frame. Advanced technologies are sought to improve the capabilities of thermionic conversion in anticipation of such requirements. Desired attributes are: improved lifetime, reasonable cost, high performance and reliability without sacrificing the basic simplicity of the technology.

\section{DISCUSSION}

Oxygenation of electrode surfaces is known to be beneficial in increasing efficiency of advanced thermionic devices because of its effect in lowering the cesiated work function of refractory 
metal surfaces. The low cesiated work function and corresponding low cesium pressure in the interelectrode space is especially of interest for pulse-mode devices in which ionization is accomplished by an applied voltage pulse.

Oxygen can be introduced deliberately at the time of sealing of the thermionic diode. Another potential source of oxygen is diffusion from the uranium oxide fuel. Unfortunately, neither method is sufficiently reliable to ensure the precise amount of oxygen required for optimum surfaces. Therefore, a means of precisely controlling the oxygen concentration in the thermionic cell is vital in realizing advanced thermionic converter performance.

A novel approach has been suggested by Desplat and Hatch et al $[2,3]$. In this approach, the actual concentration of oxygen is ignored and instead the activation potential of the oxygen is regulated, in analogy to the operation of a solid electrolyte fuel cell/electolyzer.

The use of an applied voltage pulse train to ignite the interelectrode plasma is being investigated as a means of reducing arc drop losses caused by collisional energy losses from thermionically-emitted electrons. On an energetic basis, higher efficiency results if the time averaged input pulse energy is less than the arc drop reduction, or

$$
1 / \varepsilon \int_{t=t_{1}}^{t=t_{2}} \mathrm{~V} \delta(t) d t<\left\{t_{2}-t_{1}\right\} \Delta V_{\text {arc drop }}
$$

where $\varepsilon$ is the electrical efficiency of pulse production (including transmission losses) $\mathrm{V}$ is the voltage pulse height, $\delta(t)$ is the voltage pulse shape function and

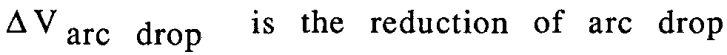
obtained by using the pulsed system as opposed to the normal non-pulsed ignited mode. For typical plasma decay times of the order $\sim 1 \mu \mathrm{sec}$, it is required that the pulse width must be of the order of .1 $\mu \mathrm{sec}$ or less.

Inductive losses are a significant concern because of the very short pulse time. For pulses to individual cells of $\sim 10$ $\mathrm{W}$ at $\sim 10 \mathrm{~V}$, inductances of even .1-1 $\mu \mathrm{H}$ are significant. This restricts the allowable geometries for thermionic converters. In particular, the flashlight cell with its relatively complex lead structure may not be practical for operations in the pulsed regime as it is understood today.

Recently, it has been suggested that the addition of trace amounts of buffer gasses such as nitrogen into the interelectrode plasma may prolong the pulse spacing and thus allow much less stringent pulse requirements [4,5]. The reason is that the gas molecules are able to store vibrational energy which is slowly released during the pulse.

Fuel swelling and resultant emitter distortion is presumed to be a potential life-limiting feature of the reactor thermionic converter. In order to combat this problem, one approach is to develop enhanced creep strength emitter materials[6]. Refractory metal alloys or even single crystal refractory metals may be used as the emitter substrate to improve the creep strength. One possibility is to fabricate the emitter by wrapping wire around a mandrel, and using chemical vapor deposition to form the rest of the emitter. A high bare work 
function material such as tungsten can be deposited over the emitter substrate.

The development of reliable cesium reservoirs remains a concern for long-lived systems. The conventional state of the art is to use intercalated graphite compounds or independently temperature-regulated two phase reservoirs for supplying the exact amount of cesium to the converter. The present approaches have problems, however. The problem with the first approach is the uncertain stability of the graphite in temperature and radiation fields for long-term operation. Distortion of the graphite from a combination of these two effects can greatly change the ability of the graphite to store cesium, thus significantly altering the operating characteristics of the thermionic converter. The problem with the second approach is that the two-phase reservoir must be separately maintained at a temperature which is typically a few hundred degrees Kelvin cooler than outlet temperature. From a spacecraft integration standpoint, it may be unacceptable to have several hundred units which must be individually instrumented and maintained. Use of a two-phase reservoir at zero-g is also an issue.

Novel approaches currently under investigation include the following: capillary-pumped reservoirs in which constant pressure is maintained due to the buffered gas heat pipe action; heavy-metal/cesium alloys which maintain a lower partial pressure of cesium than that of pure cesium; and high surface area precision loaded graphite reservoirs.

There are several reasons for investigating high temperature operating modes (operation at $2200 \mathrm{~K}$ and above.) Obviously, greatly enhanced thermionic converter performance is possible due to the greatly increased electron emission, especially when lifetime is not an overriding concern. An example is in electric propulsion applications for LEO to GEO orbit raising, in which the mission lifetime requirements may be as a few months [7]. Recent research efforts have shown that the high temperature mode can produce very high power density and efficiency for short durations [8].

Technical challenges of developing a practical converter in this regime abound. Refractory alloys capable of retaining high tensile and creep strength at high temperatures are required [9]. In addition, the vapor pressure of the emitter at these temperatures is an important consideration as well.

The inherent low-voltage, high current operating characteristic of the high temperature mode presents another potential difficulty. In order to fully realize the benefits of higher currents without prohibitive Joule losses, it is necessary to develop high electrical conductivity electrodes materials. One possible means to accomplish this is through the use of boronated graphite fibers [10]. Unlike intercalated graphite compounds, boronated graphite is thermally stable due to the substitutional bond of the boron dopants into the graphite lattice. Thus, boronated graphite acts as a p-type conductor with very high electron mobility. On a weight-normalized basis, boronated graphite can be expected to far surpass metals such as molybdenum or tungsten. There is also the added benefit of the 
superior neutronic properties of graphite compared to refractory metal. Any concern about the neutron-absorbing properties of boron can be obviated by using boron enriched in ${ }^{11} \mathrm{~B}$, which is available commercially. Other high temperature conductors can be considered as well.

\section{CONCLUSION}

Significant progress has been made since 1985 when thermionic conversion research was revitalized by renewed interest in high performance space power supplies. In particular, it is likely that advanced generations of thermionic converters will be able to achieve substantial improvements in performance while not sacrificing the basic simplicity of design which is possible in static conversion technologies. Advanced generation thermionic converters will establish a new capability in space power and provide for important new chapter in space exploration and development, in the military and the civilian sector.

\section{References.}

[1] H. J. Snyder Jr., "Star-C Space Thermionic Advanced Reactor-Compact," 1986 IECEC, p. 1320.

[2] J. L. Desplat et al., "Cesium-Oxygen Adsorption Electrode for the TRICE concept," 22nd IECEC pp. 2011-2015.

[3] G. L. Hatch et al., "Thermionic Characteristics of Planar and Cylindrical Oxygen Additive Tungsten Niobium Diodes," 22nd IECEC pp 2007-2010.
[4] V. A. Zherebetsov, "Thermionic Energy Conversion Pulse Mode," 22nd IECEC.

[5] V. A. Zherebetsov et al., "Thermionic Energy Conversion Pulse Mode with $\mathrm{N}_{2}+$ Cs," Teplofizika Visokikh Temperatur, 1984 Vol 22 No 1, p. 150.

[6] J. F. Morris and D. L. Jacobson, "Thermionic Conversion and Alloys for High Temperature Nuclear Space Power," 23rd IECEC, p. 607.

[7] E. B. Kennel and M. S. Perry, "Advanced Thermionic Nuclear Electric Propulsion for LEO to GEO Orbit Transfer in 14 Days," 22nd IECEC, p. 597.

[8] N. S. Rasor et al., "Evaluation of Thermionic Converter Performance at Very High Temperatures for Burst Power Applications," 22nd IECEC, p. 1997.

[9] J. F. Morris and D. L. Jacobson, "Material Considerations for Ultra High Temperature Thermionic Conversion for Space Nuclear Power," 21st IECEC p. 1326.

[10] M. L. Lake et al., "High Temperature Electrically Conductive Graphite Composite for Space Nuclear Power," Proceedings of the International Society ofor Optical Engineering, Vol 871, Space Structures, Power and Power Conditioning, 11-13 Jan 88. 